\title{
Bilateral anterior shoulder dislocations in bench pressing: an unusual cause
}

\author{
T R Cresswell, R B Smith
}

\begin{abstract}
A case of bilateral anterior glenohumeral dislocation in a young weight trainer is presented. The patient, an insurance clerk, had been using a free weight bar in the bench press position and had become tired when the weight on the bar forced his arms into hyperextension in the midabducted position. The humeral shaft gradually pivoted on the bench and the humeral heads were slowly dislocated anteriorly by the weight of the bar. Simple closed reduction under sedation was performed and there were no complications. After six weeks in bilateral broad arm slings, with pendulum exercises from two weeks, the patient began to mobilise his arms and he achieved a full range of movements. This unusual mechanism of injury has not previously been reported and we recommend that bench pressing should be performed with a weight that can be "locked" to prevent crushing of the user. Furthermore, the bench should be narrow enough to allow the shoulders to overhang, allowing greater extension in the abducted position without the arm pivoting on the edge of the bench. (BrF Sports Med 1998;32:71-72)
\end{abstract}

Keywords: shoulder dislocation; anterior glenohumeral dislocation; weight training; bench pressing

Bilateral glenohumeral joint dislocations, with or without fractures, in all planes are rare, as the forces required to produce a dislocation must act synchronously and in a similar manner at both joints. This most commonly occurs during epileptic convulsions when violent muscle contraction dislocates the humeral head, usually posteriorly. ${ }^{1}$ Other causes of bilateral injuries have included falls, ${ }^{2}$ nocturnal hypoglycaemia, ${ }^{3}$ electrocution, ${ }^{45}$ and even diving. ${ }^{6}$ A previous report of bilateral anterior glenohumeral dislocation in a weight lifter using the "pull over" bench movement with free weights has been described. ${ }^{7}$ Bilateral posterior dislocations may rarely also occur with fractures of the surgical neck of the humerus and often require surgical fixation. ${ }^{8-10}$ Further, bilateral anterior dislocations with fractures of the greater tuberosity have been described, ${ }^{11}$ as have bilateral anterior fracture-dislocations. ${ }^{12} \mathrm{~A}$ case of bilateral inferior dislocation has been complicated by axillary and median nerve palsies ${ }^{13}$ and another was thought to be an hysterical reaction initially. ${ }^{14}$

Bilateral simple anterior glenohumeral dislocation was reported in 1987 in a weight lifter using a bench press when, due to exhaustion, the athlete's arms were forced into extension, abduction, and external rotation. ${ }^{15}$ Never before have bilateral dislocations by the mechanism of slow levering of the arms on the edges of a bench press been reported. Fitness training is increasingly popular and is generally to be encouraged, but the use of inappropriate equipment or the untrained and unsupervised use of appropriate equipment may lead to preventable injuries.

\section{Case report}

A thirty one year old man presented to the accident and emergency department after an injury in the gymnasium. He had been using a "free weights" bar in the bench press position and had become fatigued. The weight of the bar had forced his arms, which were in mid abduction, into hyperextension. The mid shafts of the humeri pivoted on the bench edges which were wider than the shoulders. The weights caused the humeral heads to pivot forwards and dislocate anteriorly. This occurred in a slow manner. On arrival at the accident and emergency department, he was found to

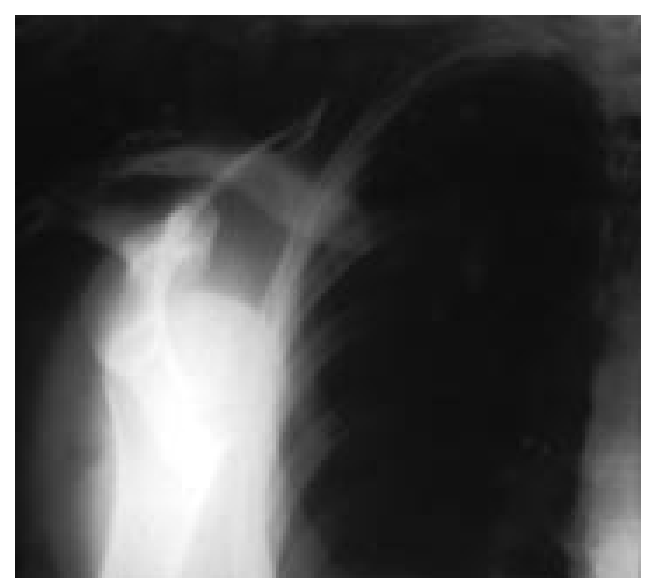

Figure 1 Radiograph of the right shoulder showing an anterior glenohumeral dislocation. 


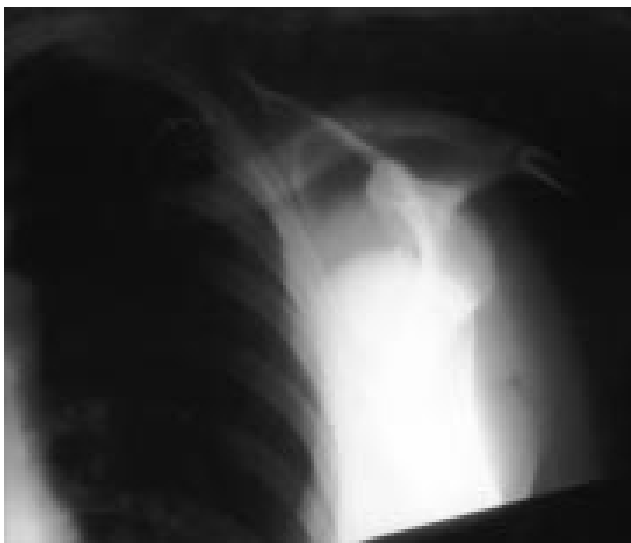

Figure 2 Radiograph of the left shoulder showing an anterior glenohumeral dislocation.

have bilateral typical anterior glenohumeral dislocations with no neurovascular deficit (figs 1 and 2), which was confirmed by anteroposterior and axillary view radiographs. He then underwent closed reduction under sedation using Kocher's method (traction, flexion, abduction with external rotation, then adduction and internal rotation). He was placed in bilateral slings for six weeks with progressive mobilisation, starting at two weeks with pendulum exercises. He made an uneventful recovery, with 0 to 160 degrees of abduction bilaterally, internal rotation to $\mathrm{T} 4$ bilaterally and 30 degrees of external rotation on the right dominant side and 40 degrees of external rotation on the left. He had no instability clinically.

\section{Discussion}

Bilateral dislocations of the glenohumeral joint in anterior, posterior, and inferior directions have been described previously in the literature for posterior dislocations and fracturedislocations, mainly after epileptic seizures. The use of gymnasium equipment is becoming increasingly popular both at home and in leisure centres and gymnasia. The danger of shoulder dislocation using a "free weights" bar in the bench press position has been described previously and can be greatly reduced if a pivoted bar with a safety lock is used. In this case the bench itself contributed to the injury in that the wide bench used allowed the humeri to pivot about the edge of the bench and thus to slowly force the humeral heads into an anterior dislocation.

We suggest that benches used for this exercise are not wider than the chest so that the shoulders are slightly overhanging and cannot therefore pivot about the bench edge allowing dislocation. Further, the weight should have a built in stop to prevent the weight falling in the event of fatigue on to the chest wall (which happened in our patient anyway). We therefore recommend that gym users are made aware of this danger and that the width of bench press benches is narrowed appropriately.

1 Thomas T, Noel E, Bouvier M. Pitfall of stiff shoulder: inveterated posterior dislocation. Clin Rheumatol 1995;14:467-70.

2 Mehta MP, Kottamasu SR. Anterior dislocation of the shoulders with bilateral brachial plexus injury. Ann Emerg Med 1989;18:589-91.

3 Litchfield JC, Subhedar VY, Beevers DG, Patel HT. Bilateral dislocation of the shoulders due to nocturnal hypoglycaemia. Postgrad Med $\mathcal{F}$ 1988;64:450-2.

4 Brown RJ. Bilateral dislocation of the shoulder. Injury 1984; 15:267-73.

5 Carew-McColl M. Bilateral shoulder dislocation caused by electric shock. Br f Clin Pract 1980;34:251.

6 Mathis RD. Bilateral shoulder dislocation: an unusual occurrence. f Emerg Med 1980;1:41-3.

7 Maffulli N, Mikhail HM. Bilateral anterior glenohumeral dislocation in a weight lifter. Injury 1990;21:254-6.

8 Marty B, Simmen HP, Kach K, Trentz O. Bilaterale anteriore Schulterluxationcfraktur nach epileptischen Anfall. Ein ore Schulterluxationcfraktur nach epileptischen An
kasuistischer Beitrag. Unfallchirurg 1994;97:382-4.

9 Page AE, Meinhard BP, Schultz E, Toledo B. Bilateral posterior fracture-dislocation of the shoulders: management terior fracture-dislocation of the shoulders: management
by bilateral shoulder hemi-arthroplasties. F Orthop Trauma

10 Martens C, Hessels G. Bilateral posterior four-part fracture-dislocation of the shoulder. Acta Orthop Belg 1995; 61:249-54.

11 Markel DC, Blasier RB. Bilateral anterior dislocation of the houlders with greater tuberosity fractures.Orthopaedics 1994;17:945-9.

12 Lal M, Yadav RS, Prakash V. Bilateral anterior fracture dislocation shoulder: two case reports. Indian f Med Sci 1992; 46:209-10.

13 Mesa M, Carpintero P, Carpintero J. Bilateral luxatio erecta humeri. Acta Orthop Belg 1996;62:116-19.

14 Brady WJ, Knuth CJ, Pirrallo RG. Bilateral inferior glenohumeral dislocation: luxatio erecta, an unusual presentation of a rare disorder. F Emerg Med 1995;13:37presen.

15 Jones M. A case of bilateral shoulder dislocation [letter]. $\mathrm{Br}$ f Sports Med 1987;21:139.

\section{Commentary}

This interesting case report outlines a potential hazard with unprotected weight lifting. This phenomenon was previously noted by Jones (as indicated in the report). As pointed out in the report, bilateral shoulder dislocations are uncommon and almost always occur posteriorly. These dislocations have been associated with seizure, electrocution, trauma, and neuromuscular disease. Simultaneous bilateral anterior dislocations present even less frequently.

The authors have clearly documented an unusual mechanism for this injury pattern. Usually, the mechanism for anterior shoulder dislocation is an extreme abnormal motion which impinges the greater tuberosity against the acromion and levers the humeral head out of the glenoid. With increased forces the impingement may cause greater tuberosity fracture. The index case is interesting in that the fulcrum for the dislocation was not the acromion, but the fixed weight bench. 